\title{
Using Hilbert Transform in Diagnostic of Composite Materials by Impedance Method
}

\author{
Volodymyr Eremenko1, Artur Zaporozhets ${ }^{2 *}$, Vitalii Babak², Volodymyr Isaienko³, \\ Kateryna Babikova ${ }^{3}$ \\ ${ }^{1}$ Department of Information Measuring Technology, Instrument Engineering Faculty, National Technical University of Ukraine \\ "Igor Sikorsky Kyiv Polytechnic Institute", 03056 Kyiv, Peremohy Avenue 37., Ukraine \\ 2 Department of Monitoring and Optimization of Thermophysical Processes, Institute of Engineering Thermophysics of NAS of \\ Ukraine, 03057 Kyiv, Marii Kapnist Street 2a., Ukraine \\ 3 Institute of Innovative Technologies and Leadership, National Aviation University, 03058 Kyiv, Ljubomyra Guzara Avenue 1., \\ Ukraine \\ *Corresponding author, e-mail: a.o.zaporozhets@nas.gov.ua
}

Received: 06 October 2019, Accepted: 25 February 2020, Published online: 13 August 2020

\begin{abstract}
The article is devoted to the problem of the increasing of information quality for the impedance method of nondestructive testing. The purpose of this article is to get for the pulsed impedance method of nondestructive testing the additional informative parameters. Instantaneous values of the information signal's amplitude is a sensitive parameter to the effects of interference, in particular friction, which necessitates the use of additional informative features. It was experimentally measured signals from defective and defectless areas of the test pattern. Using of the Hilbert transform gave possibility to determine phase characteristics of these signals and realize demodulation to extract a low-frequency envelope for further analysis of its shape. It was received the informative features as a result of researches. Among them are instantaneous frequency of a signal, the integral of a phase characteristic on the selected interval and the integral of a difference signal phase characteristics. In order to compare quality of the defect detection using selected parameters it was carried out evaluation of the testing result reliability for a product fragment made of a composite material. Considering the influence of the change in the mechanical impedance of the researched area on the phase-frequency characteristics of the output signal of the converter, it is proposed to use as the diagnostic signs: the instantaneous frequency and the value of the phase characteristic of the current signal for certain points in time. The proposed informative features enable to increase general reliability of composite materials testing by the pulsed impedance method.
\end{abstract}

Keywords

Hilbert transform, signal processing, diagnostic, defects, impedance method, signal amplitude

\section{Introduction}

Today, many industries cannot be imagined without the use of Composite Materials (CM), especially the aerospace industry. The technological process for the production of composite structural elements of an aircraft is complex and sensitive to various errors, which leads to the appearance of defects and heterogeneities in the structure of the finished product [1-3]. Often, defects in the material cause changes in the physicomechanical characteristics of the product as a whole and a decrease in its strength indicators below the maximum permissible levels. During the operation of the aircraft, its structural elements are also subject to the influence of the environment, calculated and unpredictable mechanical loads, which leads to a change in the state of the CM.

For non-destructive testing in the aircraft industry, a large number of methods are used, each of which has its own characteristics and limitations due to the types of materials being controlled and the geometric characteristics of the finished products [4-6]. One of the most common is the acoustic impedance method [7], which uses the influence of a defect to change the mechanical impedance of the system "control object - transducer". It is one 
of the main means of non-destructive testing in Europe, and is used to control sandwich panels with honeycomb, multilayer structures with polymer CM, metals and other materials and their combinations [8, 9]. The impedance method is included in the manuals for the technical operation of a number of aircraft as the basis for the periodic monitoring of cellular structures for the presence of delamination of the outer coating from the honeycomb core. Flaw detectors based on it passed interdepartmental tests, are entered into departmental registers of aviation administrations and are recommended for monitoring composite units on transport and passenger aircraft IL-76, IL-86, IL-96-300, TU-204, TU-214, Yak 42 and AN-124 «Ruslan» [10, 11]. On Airbus aircraft, the impedance method is used as an additional method for controlling elements based on CM with honeycomb core.

$\mathrm{CM}$ are characterized by discrepancies in the physical parameters and characteristics of the materials that make up the composite, a significant variation in the physical and mechanical properties both from product to product and within one product, their anisotropy, lack of sufficient information about the main types of defects, the impossibility of formally representing their models [12]. The use of traditional physical methods of non-destructive testing based on information about the physicomechanical parameters of materials, such as elastic modulus, density, ultrasonic wave propagation velocity, dielectric constant, etc., and defect models (for example, in the form of cracks, cavities, extraneous inclusions, etc.) do not fully meet the requirements for the results of diagnosing products made of composites.

The process of diagnosing is complicated by the fact that composites are characterized by a wide range of possible defects that occur both during the manufacture of products and during their operation [13-17].

At present, there is no universal physical method for diagnostics of composites, which would allow to identify all possible types of defects $[18,19]$. A change in the mechanical pulse of the interaction zone between the transducer and the control object affects the amplitude, phase, and frequency of the output electrical signal. In addition, differences in the physicomechanical characteristics of the controlled zone can lead to a change in the shape of the envelope of the information signal [20]. Typically, in the control process to determine the defect as an informative parameter, the amplitude or phase of the signal [21, 22] is used. In some cases, the spectrum of this signal is analyzed. As a rule, during controlling the impedance method, decisions about the presence of damage are made when only one parameter is exceeded. In the case of spectrum analysis, the signal energy increase in the frequency range most sensitive to the selected type of defects is taken into account. However, individual parameters (amplitude, phase, frequency) are sensitive to various disturbances, which can increase the probability of random operations. In addition, various informative parameters have different sensitivity to defects of various types.

Considering the physical features of the objects and the possibilities of obtaining primary measurement information, the most common are specially developed low-frequency acoustic methods and their modifications [23-25]. Modern devices and diagnostic systems for CM mainly use deterministic models and corresponding methods for processing informative signals and making diagnostic decisions that do not provide the necessary noise immunity and reliability of diagnostic results and defect classification [26-28].

Thus, the urgent task is to increase the number of informative parameters having a high sensitivity to possible defects.

In this work, investigations of the formation models and methods of the primary processing of information signals [29-31] will be continued.

\section{Amplitude-phase-frequency characteristics of pulsed information signals}

The information signal of an impedance transducer is an amplitude-modulated damping radio pulse whose characteristics depend on the state of the studied area [32].

The result of the Hilbert transform of a valid signal $x(t)$ defined in the time domain is Hilbert image $\tilde{x}(t)$, which also defined in the time domain. The sum of the present and received signals $z(t)=x(t)+j \cdot \tilde{x}(t)$ is the so-called "analytical" signal. Based on the analytical signal, it's impossible to obtain the amplitude bypass of the output signal $x(t)-A(t)$ and it's "instant" phase $\theta(t)$, as a function of time [33].

The Hilbert transform of arbitrary processes can be defined in three different ways.

The simplest is to define the Hilbert transform through convolution. With this approach, the Hilbert transform of a real function $x(t)$, defined in the whole domain $-\infty<t<\infty$, is a real function $\tilde{x}(t)$, given by the formula

$$
\tilde{x}(t)=H[x(t)]=\int_{-\infty}^{\infty} \frac{x(u)}{\pi(t-u)} d u .
$$

Thus, $\tilde{x}(t)$ is a convolution of the input signal $x(t)$ and the function $1 /(\pi t)$. 
Another approach is the representation of the Hilbert transform through the phase shift of the output signal $x(t)$. Let $\tilde{X}(f)$ is the Fourier transform $[34,35]$ of $\tilde{x}(t)$ :

$$
\tilde{X}(f)=F[\tilde{x}(t)]=\int_{-\infty}^{\infty} \tilde{x}(t) e^{-j^{2 \pi f t}} d t
$$

where $F[\tilde{x}(t)]$ is the Fourier transform operator.

Based on Eq. (1), $\tilde{X}(f)$ corresponds to the Fourier transform of the original function $x(t)$ multiplied by the Fourier transform of the function $1 /(\pi t)$, which is described by Eq. (3):

$$
F[1 / \pi t]=-j \operatorname{sgn} f=\left\{\begin{array}{c}
-j, f>0 ; \\
0, f=0 ; \\
j, f<0 .
\end{array}\right.
$$

Thus, Eq. (1) describes the transformation of a signal $x(t)$ by a system with a frequency response $-j \operatorname{sgn} f$ and, in turn, is equivalent of:

$$
\tilde{X}(f)=(-j \operatorname{sgn} F) X(f) \text {. }
$$

This complex function $\tilde{X}(f)$ is associated with the $\tilde{x}(t)$ by inverse Fourier transform [36]:

$\tilde{x}(t)=\int_{-\infty}^{\infty} \tilde{X}(f) e^{j^{2 \pi f t}} d f$.

The Fourier transform of the form $-j \operatorname{sgn} f$ can be represented as:

$B(f)=-j \operatorname{sgn} f=\left\{\begin{array}{l}e^{-j(\pi / 2)}, f>0 ; \\ 0, f=0 ; \\ e^{j(\pi / 2)}, f<0 .\end{array}\right.$

In this way, using Eqs. (2) and (6):

$B(f)=|B(f)| e^{-j \varphi_{b}(f)}$.

In fact, Eq. (7) describes a system that implements a phase shift $\pi / 2$ by the input signal for all positive frequencies, i.e.:

$\varphi_{b}(f)=\left\{\begin{array}{l}\pi / 2, f>0, \\ -\pi / 2 f<0 .\end{array}\right.$

If the expression $X(f)=|X(f)| e^{-j \varphi_{x}(f)}$ is transform, then:

$\tilde{X}(f)=|\tilde{X}(f)| e^{-j \tilde{\varphi}(f)}=|X(f)| e^{-j\left[\varphi_{x}(f)+\varphi_{b}(f)\right]}$.

So, the Hilbert transform is obtained by convert the signal $x(t)$ of system that leaves the module $X(f)$ unchanged, and the argument $\varphi_{x}(f)$ changes to $\varphi_{x}(f)+\varphi_{b}(f)$, where $\varphi_{b}(f)$ is defined by Eq. (8):

$z(t)=x(t)+j \tilde{x}(t)=A(t) e^{j \theta(t)}$, where $A(t)$ is the bypass of the output signal $x(t)$, and $\theta(t)$ is its instantaneous phase.

Their calculation with respect to $x(t)$ is implemented as follows, for envelope phase:

$A(t)=\sqrt{\left[x^{2}(t)+\tilde{x}^{2}(t)\right]}$,

and instantaneous phase:

$\theta(t)=\operatorname{arctg}[\tilde{x}(t) / x(t)]$.

The instantaneous frequency $f_{0}$ can be defined as the derivative of the instantaneous phase:

$f_{0}=(1 / 2 \pi) \cdot d \theta(t) / d t$

Figs. 1 to 3 present an approximate model of the information signal at the output of an impedance converter operating in pulsed mode.

The instantaneous phase $\theta(t)$ in Eq. (11) is defined on the interval $[-\pi / 2, \pi / 2]$, therefore, for calculating the phase characteristic on the interval $[0,2 \pi]$, it must be reduced to the following form:

$$
\begin{aligned}
& \Phi(t)=\operatorname{arctg}[\tilde{X}(t) / X(t)] \\
& +\pi[2-\operatorname{sign} \tilde{X}(t)(1+\operatorname{sign} X(t))] / 2+2 \pi L[X(t), \tilde{X}(t)] .
\end{aligned}
$$

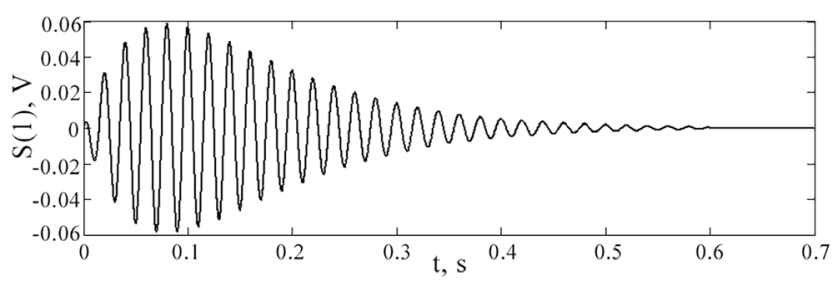

Fig. 1 Signal model in the Hilbert transform model of an amplitudemodulated damping radio pulse

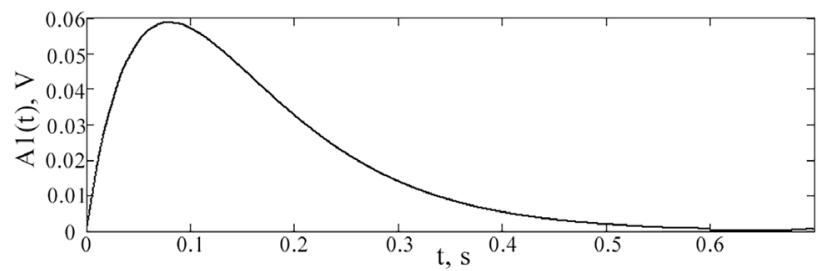

Fig. 2 The envelope of the signal in the Hilbert transform model of an amplitude-modulated damping radio pulse

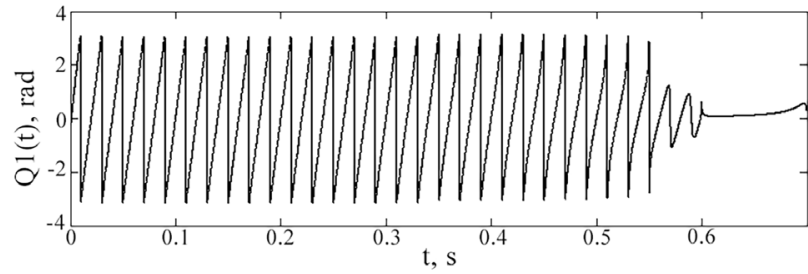

Fig. 3 The instantaneous phase of the signal during the Hilbert transform model of an amplitude-modulated damping radio pulse 
The phase shift between two signals can be determined on the basis of the so-called difference phase $Q_{2}(t)-Q_{1}(t)$. This definition allows you to operate on values within the interval $[0,2 \pi]$. To expand it, you need to go from instantaneous to a cumulative phase by complementing the expression with a step function $L[X(t), \tilde{X}(t)]$, eliminates discontinuities of the phase characteristic at points where the value of the phase characteristic reaches $2 \pi$ :

$$
\begin{aligned}
& \Phi(t)=\operatorname{arctg}[\tilde{X}(t) / X(t)] \\
& +\pi[2-\operatorname{sign} \tilde{X}(t)(1+\operatorname{sign} X(t))] / 2+2 \pi L[X(t), \tilde{X}(t)] .
\end{aligned}
$$

If the Eq. (13) make to the form:

$$
f_{0}=\Phi(t) / 2 \pi T
$$

where $T=t_{2}-t_{1}$ is the time interval at which the phase characteristic $\Phi(t)$ determined, the frequency of the carrier information signal can be determined by the rate of rise of the cumulative phase.

The easiest way to calculate cumulative phases is based on the using of Eq. (15). The phase sweep algorithm, that is, the conversion of the instantaneous phase characteristic to cumulative, realizes the elimination of gaps at points where the phase takes values greater or less than $\pi$. In case the difference between two adjacent elements of the value array exceeds $\pi$, the unwrapped phase is calculated as

$$
\Phi[i]= \begin{cases}Q[i]-\left\lfloor\frac{Q[i]-Q[i-1]}{2 \pi}+0,5\right\rfloor \cdot 2 \pi, & i=1, \ldots, N-1, \\ Q[i], & i=0,\end{cases}
$$

where $Q[i]$ is the array of samples of the instantaneous phase, $\Phi[i]$ is the array of the cumulative phase, $N$ is the length of the array; [...] is rounding operation to the nearest integer value.
Fig. 4 shows the graphs of the cumulative phases of the two information signals with carrier frequencies $f_{1}$ and $f_{2}$, differing by $10 \%$. The value of the phase difference $\Delta \Phi$ in this case depends on the period of study $T$, while the value of the instantaneous frequency remains unchanged.

From Fig. 4 it can be seen that a change in the carrier frequency of the radio pulse under consideration affects the tilt angle of the resulting Cumulative Phase Characteristic (CPC). If the frequency remains unchanged within the analysis interval, the CPC will grow monotonously. The frequency shift leads to a corresponding change in the slew rate, which allows analyzing the deviation of the carrier frequency at a given time interval.

Informative parameters calculated on the basis of $\mathrm{CPC}$ can be used as diagnostic signs when conducting diagnostics.

Figs. 5 and 6 show the implementation of the signals and graphics of their instantaneous phases with a defectfree and defective area of the test sample.

From the Figs. 5 and 6, the use of the instantaneous frequency of the signal as an informative parameter has a limitation due to the choice of the time interval on the basis of which the calculation takes place. The increase in the cumulative phase of the signal is highly non-linear, due to the non-uniformity of the deviation of the instantaneous

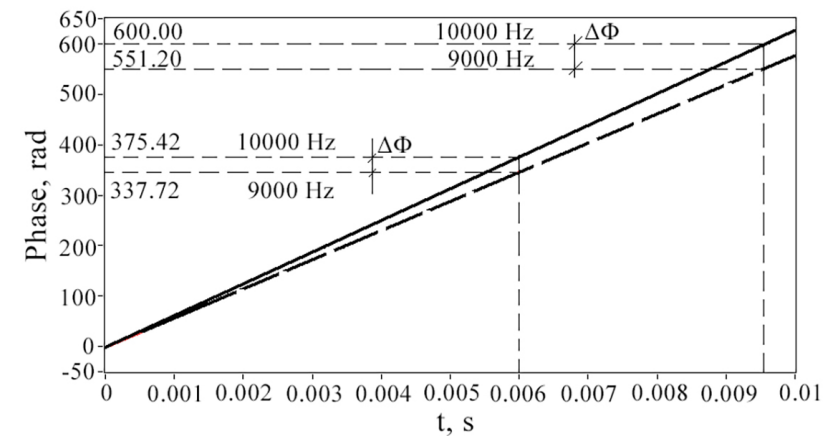

Fig. 4 The time dependences of the cumulative phases of models of information signals with different carrier frequencies

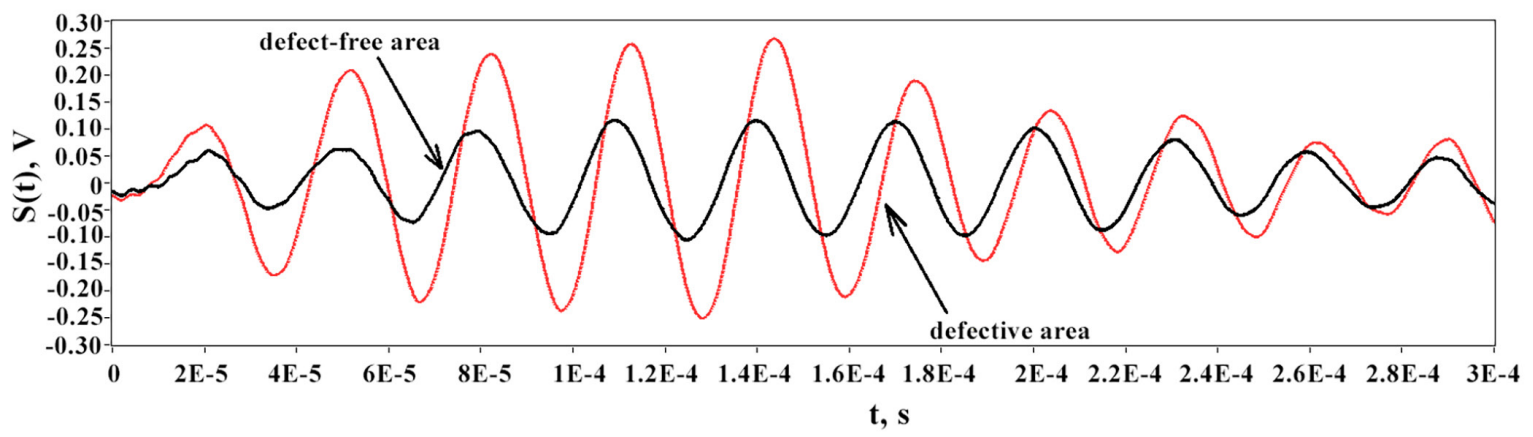

Fig. 5 Implementation of signals from defective and defect-free sample areas 


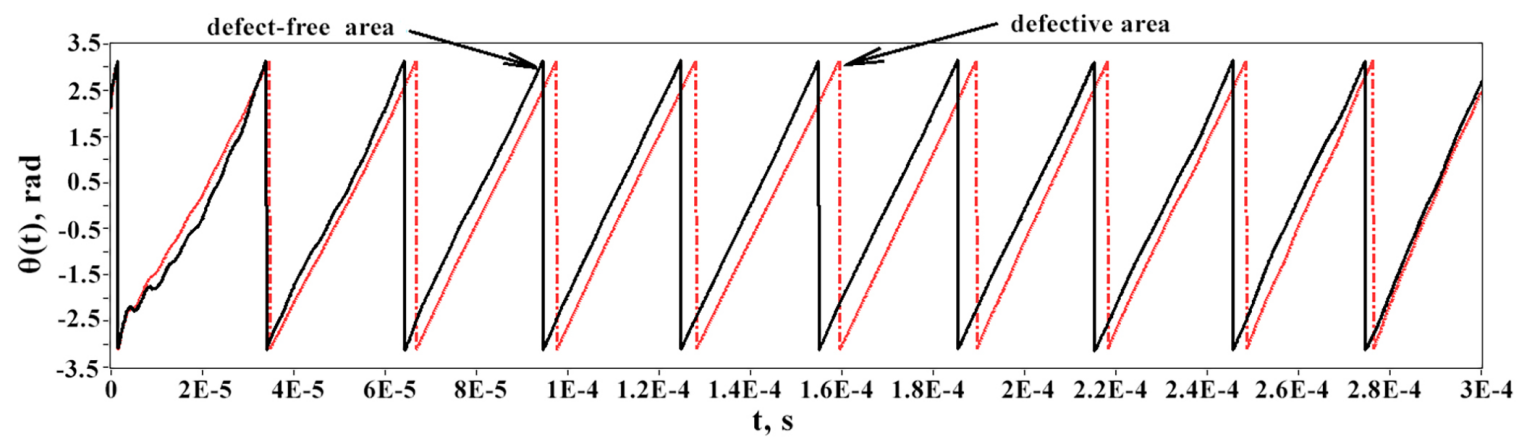

Fig. 6 Instant signal phases with defective and defect-free sample areas

phase characteristic of the current signal. The maximum deviation between implementations is observed in the time interval [0;0.00022], after which it gradually decreases. The maximum sensitivity with using the instantaneous frequency as an informative parameter will be ensured only when it is estimated at the point of the phase characteristic, which has the greatest difference in the defective and defect-free areas of the test sample. Such a situation may complicate the selection of an appropriate time interval in the process of creating a method of control.

The difference between the implementations of information signals obtained from different areas of the product can also be determined based on the study of the cumulative phase over the entire time interval of the signal.

Thus, the following research algorithm can be proposed. At the first stage, the implementation of the information signal is obtained from the defect-free region for which the reference cumulative phase characteristic $\Phi_{1}(t)$ is calculated. Next, the difference between $\Phi_{1}(t)$ and CPC obtained from the current scan area $\Phi_{2}(t)$ is calculated. The difference of the obtained characteristic will be the greater than the more significantly the physicomechanical characteristics of the controlled area differ. Fig. 7 shows the difference of CPC for defect-free and defective areas of the sample of a CM.

Based on the assumption that the transducer signal has the form of an exponentially decaying radio pulse, the analysis of information signals provides for an alternate selection of the envelope. Signal demodulation can be carried out using analog or digital methods, but at the same time, their use leads to a change in the output phase-frequency parameters of the information signal, which can cause distortions of the information characteristics embedded in it. Signal-free demodulation of the signal can be performed on the basis of the Hilbert transform discussed above. In digital systems, signal processing is performed based on its discrete representation.

The Discrete Hilbert Transform (DHT) can be obtained on the basis of the discretization of the signal, that is, the replacement of the continuous time $t$ by the defiant $n T \rightarrow n$. Then the formula (Eq. (10)) can be represented as:

$z(n)=x(n)+j \tilde{x}(n)=A(n) e^{j \theta(n)}$,

where $x(n)$ and $\tilde{x}(t)$ are the Hilbert conjugate signals; $A(n)$ is the signal envelope $x(n)$.

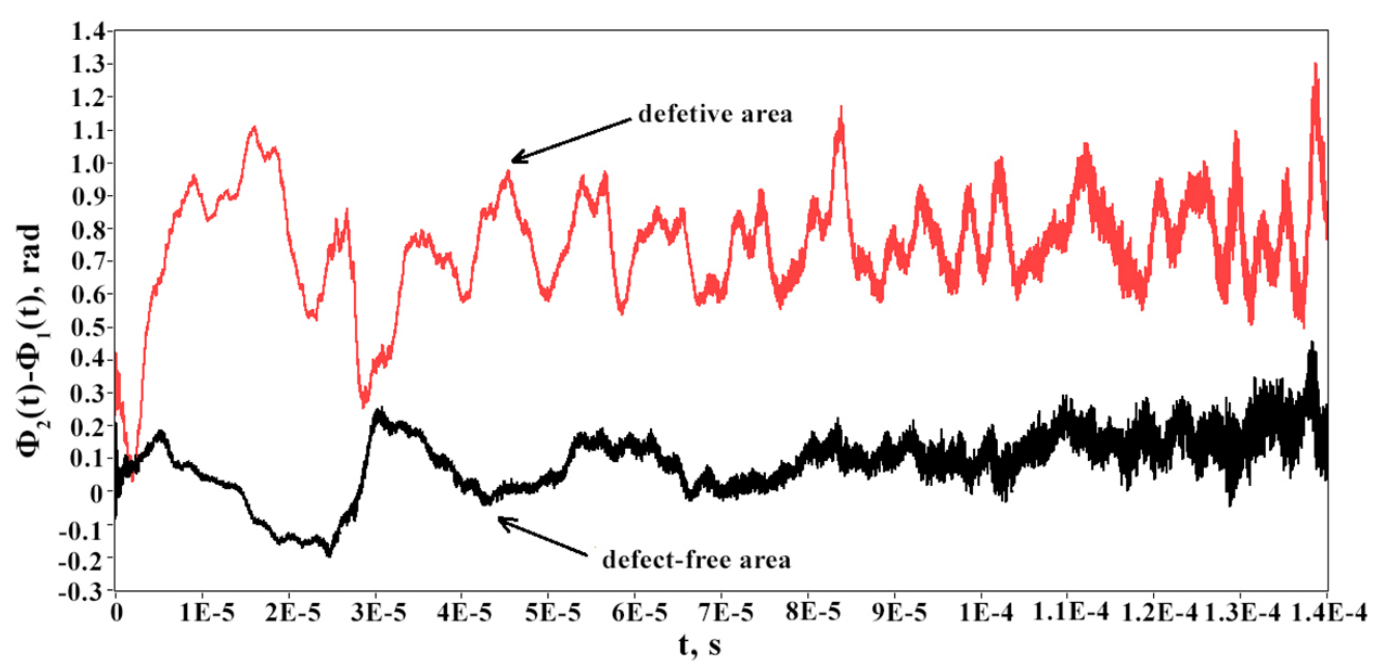

Fig. 7 Temporal representations of the difference between the reference and current implementations of CPC information signals obtained in the defective and defect-free areas 
Fig. 8 shows the bypass information signal of the transducer obtained by applying the defective and defect-free areas of the composite sample to the signals obtained by scanning with the help of a split-transducer.

From Fig. 8, the shape of the signal envelope differs significantly depending on the defectiveness of the monitored zone. At the same time, it is obvious that the signal characteristics have a variation in the middle of the zone of one class, which makes it necessary to use statistical data processing methods to highlight additional informative features based on estimates of the parameters of the random component of the results.

\section{Analysis of informative features}

To compare the diagnostic value of the proposed signs, calculated on the basis of the phase characteristics of information signals, experimental studies of various types CMs samples were carried out, had both defect-free areas and zones with damage models. Sample 1 (Fig. 9) is a honeycomb panel with a thickness of $20 \mathrm{~mm}$ with a PSP-1-2.5 type filler with plating based on ELUR-P-0.1 carbon fiber and VK 41 glue. The defect was modeled by a zone of communication failure between the plating and honeycomb. The plating on the side of the defective area showed no visual signs of damage; the skin on the opposite side was restored by sticking a patch of similar type carbon fiber.

The sample surface was scanned from the opposite zone of the skin restoration along the line that passed through the center of the defect zone. The scanning step was $3 \mathrm{~mm}$. As a mechanical impedance sensor, a separate-combined converter of the RS-1 type was used.

An experimental study was conducted as follows. At the first stage, with the help of a data acquisition system, information signal realizations were obtained. For this, the transducer was installed 30 times randomly at a point within the scan line. For each point, 5 realizations were obtained. Then, for each implementation, the values of the following diagnostic features were determined:

- signal amplitude $U_{0}$;

- instantaneous frequency $f_{0}(t)$;

- cumulative phase characteristic of the information signal $\Phi(t)$.

At the third stage, the sampling of values was averaged within the limits of each of the scanning lines, as a result of which 8 values were obtained for each attribute.

Figs. 10 to 12 present the averaged values of features that correspond to scan lines. Vertical lines indicate the boundaries of the defective areas.

On the basis of the data obtained, the statistical characteristics of the samples of diagnostic signs were calculated and are presented in Table 1.

The analysis of the effectiveness of the proposed informative features was carried out in two directions: the establishment of the relative sensitivity of the parameter to changes in the physicomechanical characteristics of the test samples in the defective and defect-free areas and stability, estimated as the average deviation at various scanning speeds.

One of the quantities affecting the accuracy of diagnosis is a measure of the dispersion of the values of the analyzed informative parameters, occurs during scanning the surface of the product. As shown above, scanning is accompanied by the effects of frictional noise on the informative signal. This leads to an increase in the dispersion of the obtained values of diagnostic signs, causes an increase in the percentage of making wrong decisions.

In order to assess the stability of the selected features during diagnostics, studies were conducted to determine their standard deviation as a function of the linear velocity of the transducer during the scanning process.

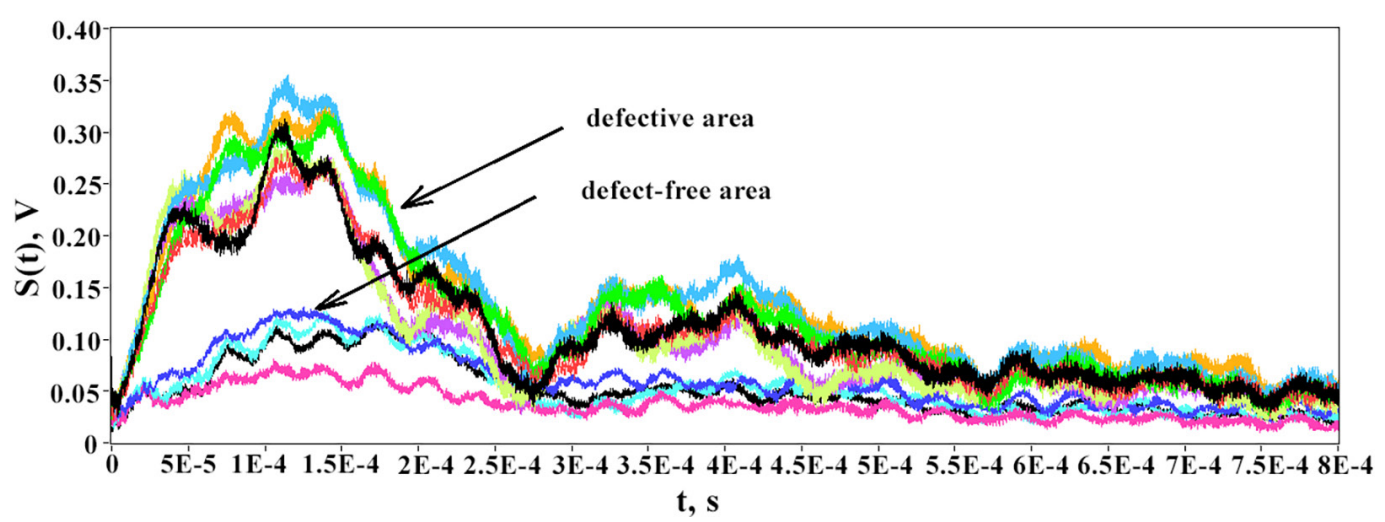

Fig. 8 Realization of internal information signals in defective and defect-free zones 


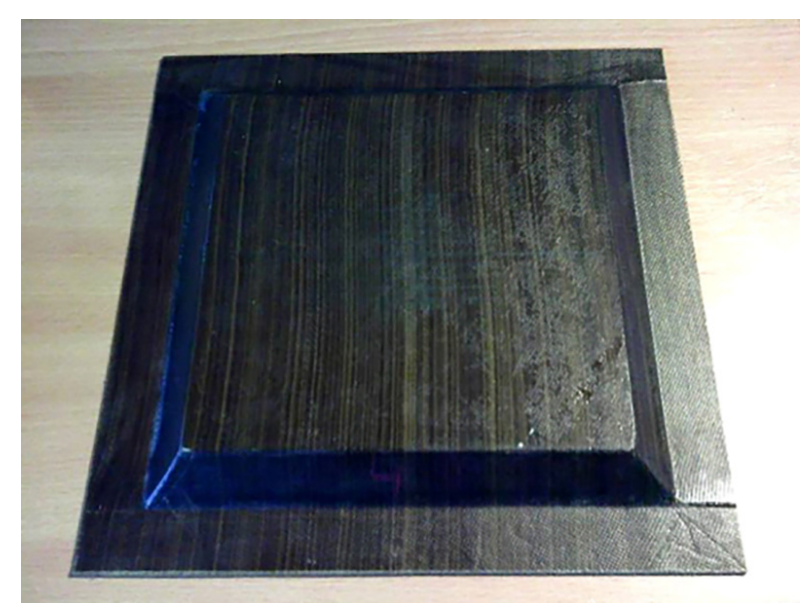

Fig. 9 Appearance of the sample

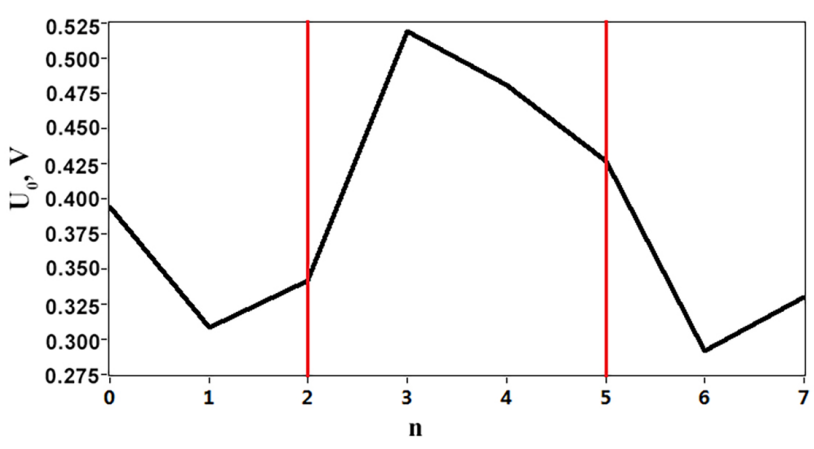

Fig. 10 Signal amplitude (implementations of informative parameter samples)

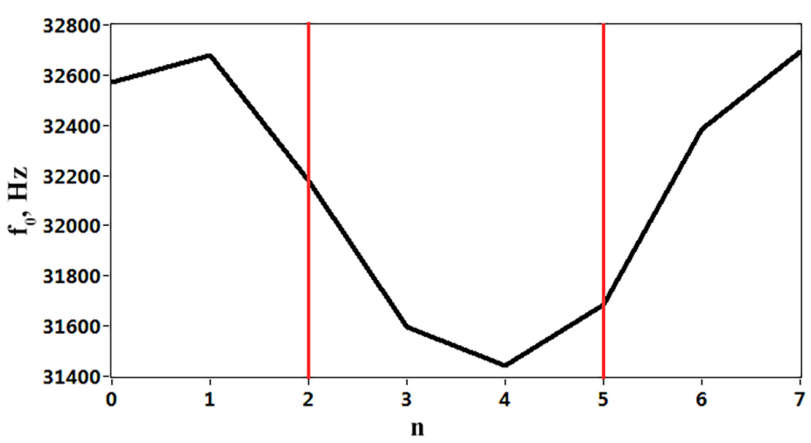

Fig. 11 Instant frequency (implementations of informative parameter samples)

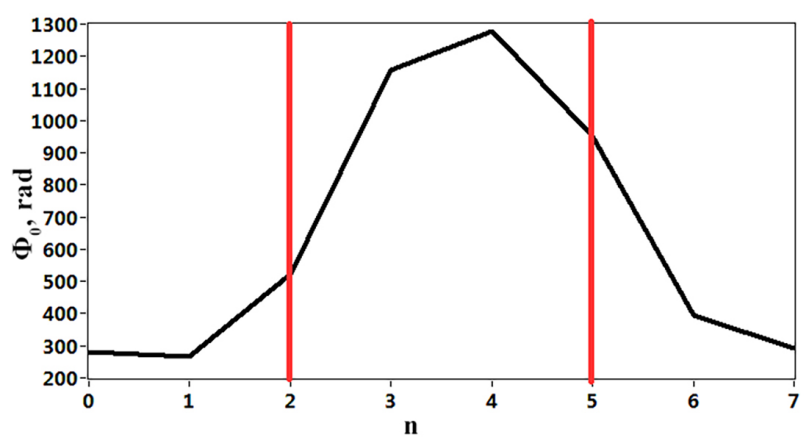

Fig. 12 Cumulative phase characteristics (implementations of informative parameter samples)
Table 1 Relative change in the statistical characteristics of informative parameterstechnical characteristics of the prototype

of the diagnosis system

\begin{tabular}{lccc}
\hline & & \multicolumn{2}{c}{ Research area } \\
$\begin{array}{l}\text { Statistical } \\
\text { characteristic }\end{array}$ & $\begin{array}{c}\text { Signal } \\
\text { parameter }\end{array}$ & Defect-free & Defective \\
\hline \multirow{2}{*}{ Expected value } & $U_{0}$ & 0.35 & 0.49 \\
& $f_{0}$ & 32695.19 & 32070.11 \\
& $\Phi_{0}$ & 297.19 & 1259.83 \\
\hline \multirow{2}{*}{ Standard deviation } & $U_{0}$ & 0.06 & 0.07 \\
& $f_{0}$ & 263.62 & 103.89 \\
Asymmetry & $\Phi_{0}$ & 209.71 & 263.57 \\
coefficient & $U_{0}$ & 0.030 & 0.037 \\
& $f_{0}$ & -0.25 & -0.04 \\
Coefficient of & $\Phi_{0}$ & 0.93 & -0.27 \\
kurtosis & $U_{0}$ & 2.31 & 2.78 \\
& $f_{0}$ & 2.54 & 2.47 \\
Confidence interval & $\Phi_{0}$ & 2.97 & 2.91 \\
for 0.95 & $U_{0}$ & $0.24-0.45$ & $0.37-0.61$ \\
& $f_{0}$ & $32261-33129$ & $32241-31898$ \\
& $\Phi_{0}$ & $48.2411-$ & $825.458-694.2$ \\
\hline
\end{tabular}

Ensuring the possibility of comparing parameters with different absolute values and scattering indices was carried out by rationing the output data. The results of the experiment are presented in Table 2.

As can be seen from above data, diagnostic signs based on an estimate of the instantaneous value of the carrier frequency of the signal and the cumulative phase are more resistant to change in the speed of movement of the converter than the amplitude ones.

\section{Conclusions}

Methods for the primary processing of information signals of acoustic diagnostic methods in time-frequency and amplitude-phase-frequency coordinates were investigated, which made it possible to carry out a structural analysis of single-pulse signals and signals with locally concentrated parameter changes and to increase the probability of diagnosis by $20 \%$.

Table 2 Relative standard deviation of diagnostic signs of samples

\begin{tabular}{cccccc}
\hline & Velocity, m/s & 0 & 1.5 & 3.0 & $>10$ \\
\hline \multirow{2}{*}{$U_{0}$} & Expected value, $\mathrm{V}$ & 0.14 & 0.15 & 0.16 & 0.20 \\
& Standard deviation & 0.093 & 0.106 & 0.114 & 0.184 \\
\hline \multirow{2}{*}{$f_{0}$} & Expected value, Hz & 32550 & 32467 & 32298 & 31030 \\
& Standard deviation & 0.008 & 0.010 & 0.010 & 0.034 \\
\hline \multirow{2}{*}{$\Phi_{0}$} & Expected value, rad & 195.51 & 162.91 & 208.56 & 506.12 \\
& Standard deviation & 0.06 & 0.08 & 0.097 & 0.118 \\
\hline
\end{tabular}


According to the experimental data obtained on the basis of analysis of variance, the degree of influence of the defect on the change of diagnostic features based on the amplitude, instantaneous frequency, and cumulative

\section{References}

[1] Gholizadeh, S. "A review of non-destructive testing methods of composite materials", Procedia Structural Integrity, 1, pp. 50-57, 2016. https://doi.org/10.1016/j.prostr.2016.02.008

[2] Jolly, M. R., Prabhakar, A., Sturzu, B., Hollstein, K., Singh, R., Thomas, S., Foote, P., Shaw, A. "Review of Non-destructive Testing (NDT) Techniques and their Applicability to Thick Walled Composites", Procedia CIRP, 38, pp. 129-136, 2015.

https://doi.org/10.1016/j.procir.2015.07.043

[3] Katunin, A., Dragan, K., Dziendzikowski, M. "Damage identification in aircraft composite structures: A case study using various non-destructive testing techniques", Composite Structures, 127, pp. 1-9, 2015.

https://doi.org/10.1016/j.compstruct.2015.02.080

[4] Zaporozhets, A., Eremenko, V., Isaenko, V., Babikova, K. "Approach for Creating Reference Signals for Detection Defects in Diagnosing of Composite Materials", In: International Conference on Computer Science and Information Technology, Lviv, Ukraine, 2019, pp. 154-172.

https://doi.org/10.1007/978-3-030-33695-0_12

[5] Ciampa, F., Mahmoodi, P., Pinto, F., Meo, M. "Recent Advances in Active Infrared Thermography for Non-Destructive Testing of Aerospace Components", Sensors, 18(2), Article Number: 609, 2018. https://doi.org/10.3390/s18020609

[6] Li, Z., Meng, Z. "A Review of the Radio Frequency Non-destructive Testing for Carbon-fibre Composites", 16(2), pp. 68-76, 2016. https://doi.org/10.1515/msr-2016-0010

[7] Jacubczak, P., Bienias, J. "Non-destructive Damage Detection in Fibre Metal Laminates", Journal of Nondestructive Evaluation, 38(2), Article Number: 49, 2019. https://doi.org/10.1007/s10921-019-0588-3

[8] Babak, V., Eremenko, V., Zaporozhets, A. "Research of diagnostic parameters of composite materials using Johnson distribution", International Journal of Computing, 18(4), pp. 483-494, 2019.

[9] Yang, J. S., Liu, Z. D., Schmidt, R., Schröder, K. U., Ma, L., $\mathrm{Wu}$, L. Z. "Vibration-based damage diagnosis of composite sandwich panels with bi-directional corrugated lattice cores", Composites Part A: Applied Science and Manufacturing, 131, Article Number: 105781, 2020.

https://doi.org/10.1016/j.compositesa.2020.105781

[10] Azarov, N., Sirbu, V. N. "Testing of bonded honeycomb structures of aircrafts by MIA based flaw detector DAMI-S", In The World of Non-destructive Testing, 3, pp. 16-29, 2003.

[11] D'Angelo, G., Palmieri, F. "Knowledge elicitation based on genetic programming for non destructive testing of critical aerospace systems", Future Generation Computer Systems, 102, pp. 633-642, 2020

https://doi.org/10.1016/j.future.2019.09.007 phase characteristic of information signals was estimated. For the amplitude, the degree of influence was $\eta_{x}=12.3 \%$, for the instantaneous frequency $-\eta_{x}=41 \%$, for the cumulative phase characteristic $\eta_{x}=46 \%$.

[12] Tam, J. H., Ong, Z. C., Ismail, Z., Ang, B. C., Khoo, S. Y. "Identification of material properties of composite materials using nondestructive vibrational evaluation approaches: A review", Mechanics of Advanced Materials and Structures, 24(12), pp. 971-986, 2017. https://doi.org/10.1080/15376494.2016.1196798

[13] Li, Z., Li, Q., Wu, Z., Yu, J., Zheng, R. "A Fault Diagnosis Method for On Load Tap Changer of Aerospace Power Grid Based on the Current Detection", IEEE Access, 6, pp. 24148-24156, 2018. https://doi.org/10.1109/ACCESS.2018.2825219

[14] Gu, F. C., Chen, H. C., Chao, M. H. "Application of improved Hilbert-Huang transform to partial discharge signal analysis", IEEE Transactions on Dielectrics and Electrical Insulation, 25(2), pp. 668-677, 2018. https://doi.org/10.1109/TDEI.2017.006922

[15] Jiao, W. "A method for time-frequency feature extraction from vibration signal based on Hilbert-Huang Transform", In: 2008 7th World Congress on Intelligent Control and Automation, Chongqing, China, 2008, pp. 8460-8464. https://doi.org/10.1109/WCICA.2008.4594607

[16] Bouden, T., Dib, S., Aissaous, K., Grimes, M. "Signal processing methods for materials defects detection", In: 2009 IEEE International Ultrasonics Symposium, Rome, Italy, 2009, pp. 1-4. https://doi.org/10.1109/ULTSYM.2009.5441482

[17] Rivas, E., Burgos, J. C., García-Prada, J. C. "Vibration Analysis Using Envelope Wavelet for Detecting Faults in the OLTC Tap Selector", IEEE Transactions on Power Delivery, 25(3), pp. 1629-1636, 2010. http://doi.org/10.1109/TPWRD.2010.2043746

[18] Xiaomeng, W., Zhang, R. "A Sensor Fault Diagnosis Method Research Based on Wavelet Transform and Hilbert-Huang Transform", In: 2013 Fifth International Conference on Measuring Technology and Mechatronics Automation, Hong Kong, China, 2013, pp. 81-84. http://doi.org/10.1109/ICMTMA.2013.32

[19] Zhao, H., Jia, J., Yang, Q., Chang, C. "Using EMD-AR spectrum for concurrent fault diagnosis of engine", In: 2014 IEEE Conference and Expo Transportation Electrification Asia-Pacific (ITEC AsiaPacific), Beijing, China, 2014, pp. 1-4. http://doi.org/10.1109/ITEC-AP.2014.6940853

[20] Bochareva, S. A., Grishaeva, N. Yu., Lyukshin, B. A., Lyukshin, P. A., Matolygina, N. Yu., Panin, S. V., Reutov, Yu. A. "A Unified Approach to Determining the Effective Physicomechanical Characteristics of Filled Polymer Composites Based on Variational Principles", Mechanics of Composite Materials, 54(6), pp. 775-788, 2019. https://doi.org/10.1007/s11029-019-9782-8 
[21] Babak, S., Babak, V., Zaporozhets, A., Sverdlova, A. "Method of statistical spline functions for solving problems of data approximation and prediction of objects state", In: 7th Workshop on Cloud Technologies in Education (CTE 2019), Kryvyi Rih, Ukraine, 2019, pp. 810-821. [online] Available at: http://ceur-ws.org/Vol2353/paper64.pdf [Accessed: 01 May 2019]

[22] Galagan, R. M., Momot, A. S. "The use of ART-2 neural network for processing information signals of non-destructive testing", In: 2017 IEEE First Ukraine Conference on Electrical and Computer Engineering (UKRCON), Kiev, Ukraine, 2017, pp. 981-985. http://doi.org/10.1109/UKRCON.2017.8100395

[23] Babak, V., Filonenko, S., Kalita, V. "Acoustic emission under temperature tests of materials", Aviation, 9(4), pp. 24-28, 2005. https://doi.org/10.3846/16487788.2005.9635914

[24] Babak, V., Filonenko, S., Kalita, V. "Research of acoustic emission signals at destruction of graphite", Aviation, 10(1), pp. 10-16, 2006. https://doi.org/10.3846/16487788.2006.9635921

[25] Babak, V., Filonenko, S., Stadnychenko, V. "Mechanism of transition of friction pairs to "quasiwearless" mode of operation", Aviation, 10(4), pp. 8-13, 2006. https://doi.org/10.3846/16487788.2006.9635941

[26] Li, H., Zheng, H., Tang, L. "Gear Fault Diagnosis Based on Order Tracking and Hilbert-Huang Transform", In: 2009 Sixth International Conference on Fuzzy Systems and Knowledge Discovery, Tianjin, China, 2009, pp. 468-472. https://doi.org/10.1109/FSKD.2009.220

[27] Rivera-Calle, F. M., Minchala-Ávila, L. I., MontesdeocaContreras, J. C., Morales-Garcia, J. A. "Fault diagnosis in power lines using Hilbert transform and fuzzy classifier", In: 2015 International Conference on Electrical Systems for Aircraft, Railway, Ship Propulsion and Road Vehicles (ESARS), Aachen, Germany, 2015, pp. 1-5. http://doi.org/10.1109/ESARS.2015.7101420

[28] Goharrizi, A. Y., Sepehri, N. "Internal Leakage Detection in Hydraulic Actuators Using Empirical Mode Decomposition and Hilbert Spectrum", IEEE Transactions on Instrumental and Measurement, 61(2), pp. 368-378, 2012. http://doi.org/10.1109/TIM.2011.2161938
[29] Zaporozhets, A. "Analysis of Control System of Fuel Combustion in Boilers with Oxygen Sensor", Periodica Polytechnica Mechanical Engineering, 63(4), pp. 241-248, 2019. https://doi.org/10.3311/PPme.12572

[30] Zaporozhets, A., Eremenko V., Serhiienko, R., Ivanov, S. "Methods and Hardware for Diagnosing Thermal Power Equipment Based on Smart Grid Technology", In: Conference on Computer Science and Information Technologies (CSIT 2018), Lviv, Ukraine, 2018, pp. $476-489$

https://doi.org/10.1007/978-3-030-01069-0_34

[31] Maanani, Y, Menacer, A. "Modeling and Diagnosis of the Inter-Turn Short Circuit Fault for the Sensorless Input-Output Linearization Control of the PMSM", Periodica Polytechnica Electrical Engineering and Computer Science, 63(3), pp. 159-168, 2019. https://doi.org/10.3311/PPee.13658

[32] Cawley, P. "The sensitivity of the mechanical impedance method of nondestructive testing", NDT International, 20(4), pp. 209-215, 1987. https://doi.org/10.1016/0308-9126(87)90243-4

[33] Eremenko, V., Zaporozhets, A., Isaenko, V., Babikova, K. "Application of Wavelet Transform for Determining Diagnostic Signs", In: 7th Workshop on Cloud Technologies in Education (CTE 2019), Kryvyi Rih, Ukraine, 2019, pp. 202-214. [online] Available at: http://ceur-ws.org/Vol-2387/20190202.pdf [Accessed: 25 June 2019]

[34] Babak, V., Mokiychuk, V., Zaporozhets, A., Redko, O. "Improving the efficiency of fuel combustion with regard to the uncertainty of measuring oxygen concentration", Eastern-European Journal of Enterprise Technologies, 6(8), pp. 54-59, 2016. http://doi.org/10.15587/1729-4061.2016.85408

[35] Zaporozhets, A. O., Redko O. O., Babak, V. P., Eremenko, V. S., Mokiychuk, V. M. "Method of indirect measurement of oxygen concentration in the air", Naukovyi Visnyk Natsionalnoho Hirnychoho Universytetu, 5, pp. 105-114, 2018. https://doi.org/10.29202/nvngu/2018-5/14

[36] Zheng, J., Su, T., Zhu, W., He, X., Liu, Q. H. "Radar High-Speed Target Detection Based on the Scaled Inverse Fourier Transform", IEEE Journal of Selected Topics in Applied Earth Observations and Remote Sensing, 8(3), pp. 1108-1119, 2015. https://doi.org/10.1109/JSTARS.2014.2368174 